\title{
Application of silver needle thermal therapy in the chronic pain
}

\author{
Junmin Yu, Chuansheng Liu, Yanwei Yin, Fenghe Xu, Yusheng Yi and Chao Meng* \\ Department of Pain Management, The Affiliated Hospital of Qingdao University, Qingdao, China
}

\begin{abstract}
Silver needle thermal therapy (SNT) originated in the 1970s. Initially, SNT was created through combining muscle relaxation and acupuncture treatment basing on human soft-tissues anatomy by Prof. Renzhe Xuan, and applied this technique to the treatment of intractable chronic soft-tissues pain. It has been achieved good therapeutic effects. With the development of technology, especially the temperature-controlled silver needle therapy instrument instead of burning cotton ball, SNT technology is further promoted in the clinic, and widely used in the treatment of chronic pain. This is a review about the progress and development of SNT and its application in chronic pain.
\end{abstract}

\section{Introduction}

The silver needle contains $85 \%$ silver and a small amount of copper, chromium, nickel and other metals, thus provides the good thermal conductivity of the needle and ensures the elasticity and toughness of the needle during the puncture process to avoid excessive tissue damage or breakage of needle [1]. Initially, the diameter of silver needle is $1.1 \mathrm{~mm}$, and the length has different specifications: $11 \mathrm{~cm}, 13 \mathrm{~cm}, 15 \mathrm{~cm}$, and $17 \mathrm{~cm}$. Each silver needle has a needle handle $(5 \mathrm{~cm})$ using to connect the temperature-controlled silver needle therapy instrument (YW-L1000; SHANXI ASTRONANTICS IN GOAL DIRECTION MED TECH Co., LTD., China). According to the thickness of soft-tissue in different parts, different lengths of silver needles are selected. Recently, with increasing treatment for relatively thin soft-tissues such as internal and external collateral ligament of knee and plantar fasciitis, silver needle added categories: a silver needle with $0.45 \mathrm{~mm}, 0.6 \mathrm{~mm}$, and $0.8 \mathrm{~mm}$ diameter, $5.5 \mathrm{~cm}, 8 \mathrm{~cm}$, and $6 \mathrm{~cm}$ needle body length and $5 \mathrm{~cm}, 3 \mathrm{~cm}$, and $3 \mathrm{~cm}$ needle handle, respectively (Figure 1).

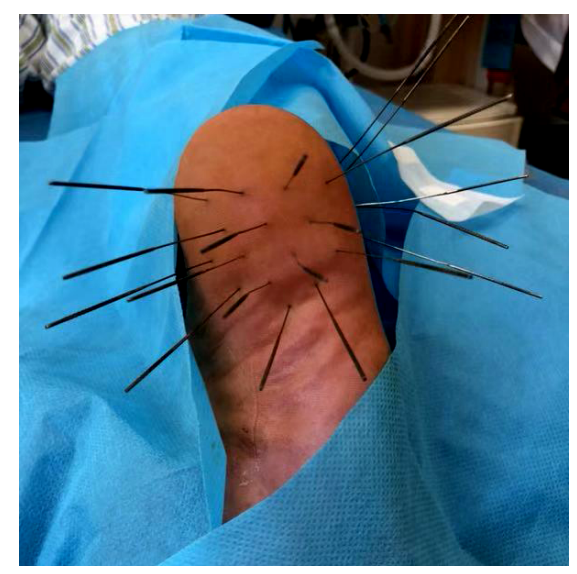

Figure 1. Silver needle thermal therapy for knee arthritis. The needles are punctured obliquely around the medial and lateral collateral ligament
The temperature-controlled silver needle therapy instrument is used to heat the silver needle handle and conduct to the needle body in order to increase the temperature of surrounding area of the needle. Different sizes of silver needles need to be equipped with different temperature-controlled silver needle therapy instruments. Currently, there are 2 common types of temperature-controlled silver needle therapy instrument: one for $3 \mathrm{~cm}$ length of needle handle and one for $5 \mathrm{~cm}$.

\section{Silver needle thermal therapy}

The position, direction, and number of the needles during the silver needle thermal therapy (SNT) are all based on the targeted muscle and the anatomy of soft-tissues. SNT is an invasive treatment and the whole operation process should follow the principle of sterility.

Before the SNT, we should complete the auxiliary examination to confirm the diagnosis and the targeted parts. According to the distribution of the corresponding muscles in targeted parts, the needled range and the puncture point are determined. The scope of disinfection should be $15 \mathrm{~cm}$ around the targeted area (Figure 2).

Fugen Wang [2] found that the needle temperature closed to the skin was $43-45^{\circ} \mathrm{C}$, and the needle tip was about $39^{\circ} \mathrm{C}$ when the silver needle was heated. Clinically, it was found that a circular "erythema" with a diameter of 1-1.2 cm centered on puncture point was formed after heating, which was the range of soft-tissues affected. The influencing factors include the length of silver needle, the setting temperature of the

${ }^{*}$ Correspondence to: Chao Meng, Department of Pain Management, the Affiliated Hospital of Qingdao University, Qingdao, China, Tel: +86-18661808786, E-mail: mengchao1119@126.com

Key words: silver needle thermal therapy, temperature-controlled, silver needle therapy instrument, chronic pain

Received: November 05, 2018; Accepted: December 14, 2018; Published: December 20, 2018 


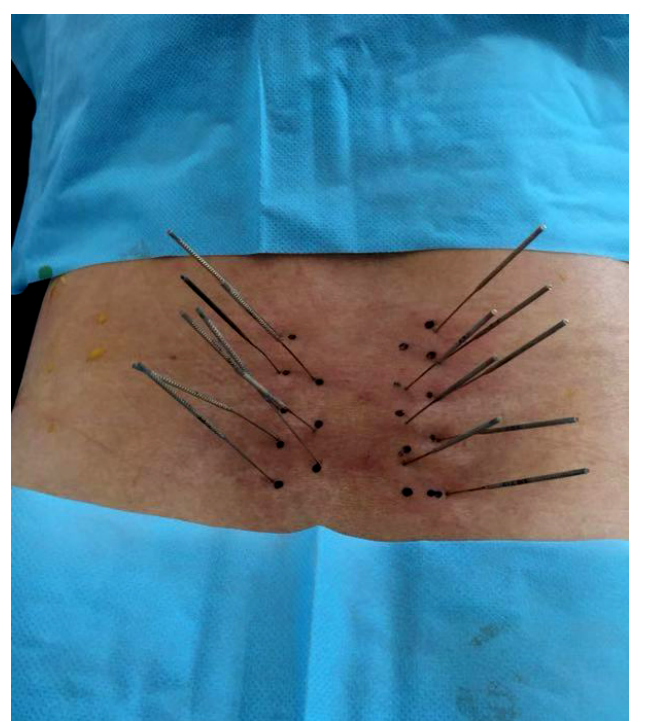

Figure 2. Silver needle thermal therapy for disc diseases. The needles are punctured into laminar and outer intervertebral foramen

temperature-controlled silver needle therapy instrument, and the room temperature. Generally, the interval of each silver needle is $1.5-2 \mathrm{~cm}$, and every three-silver needle is best to form an equilateral triangle in order to increase the covered area and decrease the using silver needles. Silver needles are suggested to puncture to the bone surface and set the temperature to $80^{\circ} \mathrm{C}-120^{\circ} \mathrm{C}$ according to the length of outside silver needle. The most important is to be careful to avoid touching the nerves. The heating time is $15-25 \mathrm{~min}$.

A local skin wheal created using $1 \%$ lidocaine could not completely alleviate the pain during the process of silver needle puncture, and local anesthesia itself also causes pain. Nerve block anesthesia, local anesthesia, and basic anesthesia are also alternative anesthesia. However, whether anesthesia and which anesthesia method chosen has not reached consensus, and there are no relative researches.

When the heating finished, the silver needles are pulled out after the temperature of the needle handle lowered. After pressing to stop hemorrhage, disinfection of skin with iodophor is performed. The patients should keep the puncture sites dry within 3 days to avoid infection. The muscle biomechanics will be changed in the soft-tissue of treatment sites within 2 months after SNT [3]. So, the repeated treatment in the same parts is usually after 2 months.

\section{Mechanisms of SNT}

\section{Elimination of the sterile inflammation in soft-tissues}

The injury and repair of skeletal muscle are a very complicated process. During this period, various inflammatory factors such as interleukin (IL) and kinins lead to a series of aseptic inflammatory reactions to induce pain. Some studies [4,5] found that IL-8 and IL-6 were decreased after SNT in a model of rabbit and rat skeletal muscle injuries, which demonstrated the mechanism of SNT was associated with the decreased inflammation factors. Therefore, reducing inflammation reaction is one of the reasons of SNT to relieve pain.

\section{Improvement of the blood circulation of soft-tissues}

Skeletal muscle injury could induce skeletal muscle fiber connective tissue hyperplasia to affect the number of blood vessels. Clinically, infrared thermography shows that the skin temperature of the diseased area is significantly lower than the normal area. Fugen Wang [6] found that the blood flow increased by $50-150 \%$ in the deep muscles of patients with soft-tissues pain after SNT. Wenyu Zhang [7] found that SNT can effectively increased the content of vascular endothelial growth factor (VEGF) and promote the repair of skeletal muscle injury. Gang Wang [8] also demonstrated that SNT improved the microcirculation of injured gastrocnemius muscle in a rat model. Therefore, SNT reduced the soft-tissues pain through improving the blood circulation.

\section{Relief of muscle spasm}

Yongzhi Wang [9] found that compression increased the muscle tone, SP release, and reduced mechanical pain threshold in a rat model of Ganz compression. Delong Wang [10] found that there was a clear relevance between local muscle spasm and the pain of the corresponding part in patients. We also found that patients with unilateral chest and back pain often had higher muscle tone, and SNT improved the softtissues tension. Jing Xiao [3] thought that SNT improved the muscle tone of erector spinae in the patients continuously from 1 week to 3 months. Gang Lu [11] also found that SNT reduced the muscle spasm and improved the quality of life in patients with lumbar disc herniation. Therefore, Relief of muscle spasm is also a mechanism of SNT.

\section{SNT application in the clinical chronic pain}

\section{Skeletal muscle diseases}

Presently, SNT is more widely used in refractory skeletal muscle pain in the clinic. SNT attenuated the pain of neck and shoulder and improved the quality of life in patients [12]. It was also demonstrated that SNT provided significantly therapeutic effects in the fasciitis of lower back, neck, and planta [13-15]. For the patients with piriformis syndrome induced back and hip pain and sciatica, SNT not only decreased the pain but also improved the recovery of piriformis tension and functional $[16,17]$. For many skeletal muscle diseases, SNT could be an effective treatment.

\section{Disc diseases}

SNT is one of the important ways of conservative treatment of disc disease. SNT reduced the spontaneous electrical activity [18] and increased the $\beta$-EP (endorphin) activity to achieve good immediate and long-term analgesic effects in patients with lumbar disc herniation (LDH) [19]. A meta-analysis showed that SNT provided better therapeutic effects than other conservative treatments for LDH patients in the short-term and middle-term (within 6 months) [20]. Yongguo Ding found SNT also alleviated pain induced by recurrence of patients with LDH [21]. So, SNT also has a therapeutic effect on the disc disease.

\section{Neuropathic pain}

SNT's effects of elimination of aseptic inflammation and improvement of microcirculation may reveal that SNT could provide therapeutic effects on neuropathic pain, which has been demonstrated in many studies. Mingjian Zhou [22] found that SNT relieved local tissue spasm and pain symptom for patients with hip epithelial neuralgia. SNT also significantly relieved the patient's occipital neuralgia [23] and post-herpetic neuralgia [24], which was better than single use of radiofrequency or block therapy. Therefore, SNT could be used for neuropathic pain (Figure 3). 


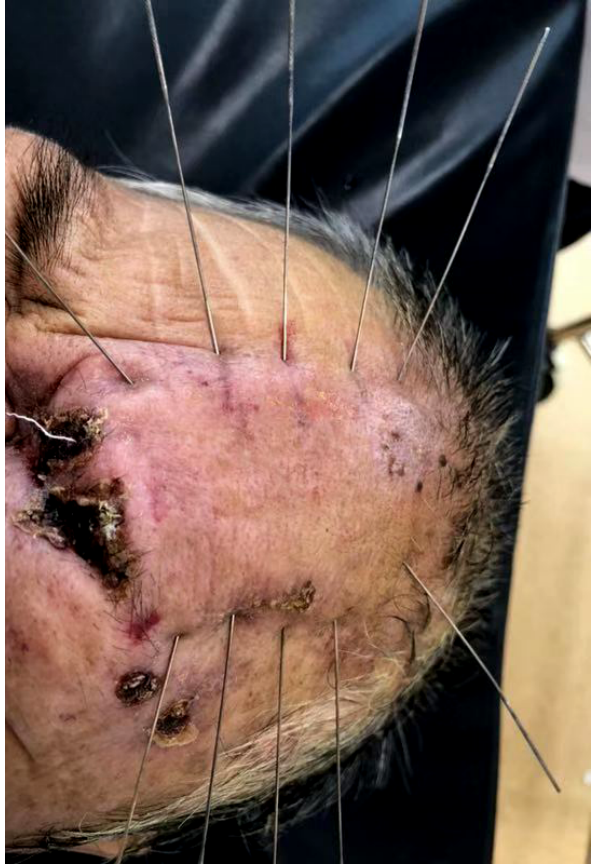

Figure 3. Silver needle thermal therapy for neuropathic pain. For post-herpetic neuralgia in left forehead, the needles are punctured obliquely to cover the pain area

\section{Conclusion}

In 2001, pain was listed as the fifth vital sign, and the treatment for chronic pain is increasingly valued. As an important method for chronic pain, SNT can clearly alleviate the pain of patients with skeletal muscle diseases, disc diseases, and neuropathic pain, promote the function recovery, and improve the quality of life. However, the SNT's specific mechanisms and the more evidence for clinical application need to be studied in the future. We believe that SNT deserves further clinical promotion.

\section{Conflicts of interest}

We declare that none of the authors have any financial conflicts of interest that may influence this manuscript.

\section{References}

1. Ning H, Wang Y, Yuan Y, Ning H (2015) Nursing and safety of silver needle diathermy treating ankylosing spondylitis. Pak J Pharm Sci 28: 725-729. [Crossref]

2. Wang F, Jiang Y, Feng C, Fu Q, Hou J, et al. (2005) Clinical study of silver needle thermal therapy. Chinese J Pain Med 11: 5-6.

3. Xiao J, Zhu L, Jin T, Chen J, Yu J, Feng M (2014) Comparison of paravertebral soft tissue tension changes in patients with chronic lower back pain treated by sliver needle and traditional Chinese medicine fumigation. Zhongguo Gu Shang 27: 513-517. [Crossref]

4. Xie C, Liu B, Ding Q, Ding Z, Zhou Q, Liu Y (2014) Effect of silver needle thermal therapy on the expression of interleukin-6 in achilles tendinopathy rats. J Rehab Med 29: 1079-1081.

5. Feng C, Chen H, Wang F, Gao Q, Wang J, Hou J (2005) Effect of warmed silver needle therapy on the level of interleukin- 8 in skeletal muscle of rabbit with chronic quadriceps injury. Chinese J Clin Rehabil 9: 98-99.

6. Wang F, Fu Q, Hou J, Wang K, Wang F, et al. (2001) Study on the changes of the volume of blood flow before and after silver needle acupuncture treatment in patients with extravertebral soft tissue lesion. Chinese J Pain Med 7: 80-82.

7. Zhang W, Gao Q, Wang G, Wang F, Yang Z (2012) Effects of internal heated needle on VEGF and BFGF in the chronic injury tissue in rats. $J$ Health Care Med 14: 193-196.
8. Wang G, Gao Q, Wang Z, Wang F (2011) Effect of different acupuncture therapy on microvascular perfusion after blunt skeletal muscle injury in rats. Academic Journal of PLA Postgraduate Medical School 32: 651-653.

9. Wang Y, Guo C, Zhong H, Wang D, Wang X (2012) Study on the effects of soft tissue compression on muscle tension, mechanical pain threshold, and substance $\mathrm{P}$ in rats. Beijing Journal of Traditional Chinese Medicine 31: 923-926.

10. Su Y, Dong F, Wang D, Wang X (2014) Effect of Pi-Zhen on 5-HT content in nerve endings tension pain rabbit model. World Science and Technology: Modernization of Traditional Chinese Medicine 6: 1256-1260.

11. Lu G, Yuan X, Hu Y (2013) Clinical observation of silver needle in the treatment of lumbar disc herniation. Ningxia Medical Journal 35: 1086-1087. [Crossref]

12. Xu W, Xie L, Jia J, Gu J, Hong Y (2015) Therapeutic effect of intensive silver needle on 40 cases of cervical spondylosis. Yunnan Journal of Traditional Chinese Medicine 36: $48-50$.

13. Zhao J, Peng L, Tang C (2018) Clinical effect of fine silver needle on lumbar myofasciitis and its influence on autonomic nerve function. Practical Journal of Medicine \& Pharmacy 35: 709-711.

14. Ou L, Che W, Cao H, Chen Z, Chen X (2017) Therapeutic effect of heat treatment with internal heat and silver needle on fasciitis. Guangxi Journal of Traditional Chinese Medicine 40: 24-26.

15. Chen L, Shang H, Lu H (2017) Clinical study on treatment of low back muscle fasciitis with silver needle. Massage and rehabilitation medicine 8: 23-24.

16. Gu N, Tang C (2015) Intensive silver needle lysis combined with ultra-laser therapy for piriformis syndrome. Practical Journal of Medicine \& Pharmacy. 5: 421-422.

17. Wang G, Liu L, Wang S, Wang B (2015) Clinical observation on piriformis syndrome by silver needle heat moxibustion. Journal of Liaoning University of Traditional Chinese Medicine 17: 13-14.

18. Wang F, Jiang Y, Wang S (1999) Electromyography before and after silver needle acupuncture treatment in patients with protrusion of the lumbar intervertebral disc. Chinese Journal of Pain Medicine 5: 194-197.

19. Zhang Q, Guo C (2012) Analgesic effect of silver acupuncture and its influence on level of serum $\beta$-Ep in patients with lumbar disc herniation. Chinese Journal of Rehabilitation. 27: $342-344$.

20. Wang Y, Leng P, Mu B, Li X, Zhang H (2016) Safety and therapeutic evaluation of silver needle therapy treating lumbar disc herniation. Chinese Archives of Traditional Chinese Medicine 34: 2737-2740.

21. Ding Y, Zhen L, Zhang C, Gu J, Jia J, et al. (2013) Treatment of 28 cases of postoperative recurrence of lumbar disc herniation with silver needle. Chinese Journal of Pain Medicine 8: 511-5112.

22. Zhou M, Wang J, Kou M, Liu D (2011) Clinical research about treating pain in superior clunial nerves through paroxysmal pulling manipulation of fixed-point unite silver needle. China Practical Medical 6: 10-12.

23. Zhang H, Yuan T (2013) Observation on treatment for suboccipital triangle syndrome with intensive acupuncture. Gansu Journal of Traditional Chinese Medicine 26: 100101.

24. Liu T, Huang J (2018) Clinical observation of Yiqi Tongluo decoction combined with silver needle thermal therapy for middle-aged post-herpetic neuralgia. Modern Journal of Integrated Chinese Traditional and Western Medicine 27: 2325-2328.

Copyright: (C2018 Yu J. This is an open-access article distributed under the terms of the Creative Commons Attribution License, which permits unrestricted use, distribution, and reproduction in any medium, provided the original author and source are credited. 